\section{Extreme High-Resolution SEM: A Paradigm Shift}

Richard Young*, Todd Templeton*, Laurent Roussel ${ }^{* *}$, Ingo Gestmann $n^{* *}$, Gerard van Veen ${ }^{* *}$, Trevor Dingle ${ }^{\star *}$ and Sander Henstra ${ }^{* *}$ FEI Company, ${ }^{*}$ Hillsboro, OR and ${ }^{*}$ Eindhoven, The Netherlands Richard.young@fei.com

"Extreme high-resolution" (XHR) scanning electron microscopy (SEM) has the potential to change the way we look at SEM. Anyone in the SEM world knows that you don't do high-resolution SEM at low accelerating voltages because of chromatic aberration limitations. The XHR design offers a new way to deal with chromatic aberration and realize the huge benefit of reduced beam penetration.

The new Magellan 400 SEM family is the first to offer subnanometer resolution over the entire electron energy range from 1 $\mathrm{keV}$ to $30 \mathrm{keV}$, effectively establishing a new performance category known as XHR SEM (Figure 1). To achieve this unprecedented performance, the Magellan combines novel electron optical design elements with technologies developed for the industry-leading Titan (scanning) transmission electron microscope (S/TEM) and DualBeam (focused ion /SEM) platforms.

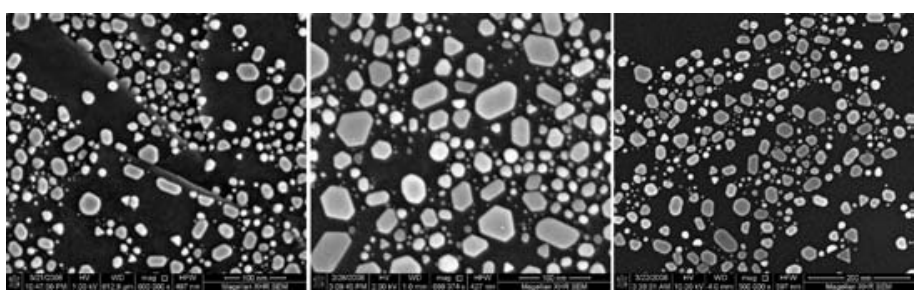

Figure1: Gold on carbon, the original images were analyzed for resolutionconfirming<0.9nm@1 kV(a),<0.8nm@2kV(b), <0.8nm@ $10 \mathrm{kV}(\mathrm{c})$. Horizontal field widths (HFW): 500, 430, $600 \mathrm{~nm}$ respectively.

\section{Unique Capability}

The system's extraordinary low-voltage performance provides high-resolution, surface-specific information that is simply unavailable from other techniques. Although SEM is considered a surface imaging technique, it would be more accurately called near-surface since the signals it uses may originate as much as a micrometer below the surface, depending upon the beam energy used. S/TEM images, in contrast, are projections presenting information generated as the $200-300 \mathrm{kV}$ electrons travel through a thin section. They can reveal detailed internal structure at atomic resolution, and in some cases, using advanced three-dimensional (3D) tomography reconstruction techniques, some detail about internal surfaces and interfaces. The XHR SEM's low-voltage capabilities allow it to provide exquisitely-detailed images of complex 3D surfaces that are difficult or impossible to obtain any other way.

\section{XHR Applications}

A key driver for the development of the XHR SEM came from the semiconductor market. Whether for research, process development, process control, or for failure analysis, semiconductor device manufacturers have made heavy use of SEMs throughout the history of their industry. With new processes being introduced at design rules of $45 \mathrm{~nm}$ and below, the drive for high-resolution information of their ever smaller layers and structures pushes them in two main directions - lower beam energies in the SEM (to allow for more surface sensitive imaging) and to more S/TEM analysis.
Each technique has its own benefits - SEM generally has simpler sample preparation, and lends itself to high-throughput imaging of cleaved sections and of complex 3D surfaces, while S/TEM offers atomic level imaging and chemical analysis, unmatched by SEM, but requires much more sample preparation and is not well suited to inspecting 3D surface features. Therefore, it is to be expected that both XHR SEM and S/TEM will become more prevalent and critical with the progression to each new, smaller technology node.

Figure 2 shows an XHR SEM application, where a deprocessed sample has been stripped back to the poly-silicon level. Here the benefits of using XHR SEM to inspect the complex 3D surfaces created are apparent - the sample can be navigated around at lower magnification (Fig. 2a) and then areas of interest can be zoomed into (Fig. 2b and c) to observe the intersection of various layers. The flexibility of the XHR SEM to tilt and rotate enables multiple viewpoints to be used. With the advent of FIN-FET and similar $3 \mathrm{D}$ gate structures in future nodes, these kinds of needs will only increase. Similarly, SEMs have historically been used to inspect multiple cleaved samples in cross-section, or image samples top down (Fig. 3). To achieve the throughput needed for these applications, it is important to be able to mount multiple samples into the system and to avoid having to perform excessive sample preparation just to make the sample small enough to fit onto the stage.

Just as semiconductor and data storage manufacturers are well into the nanotechnology realm, the research community is also driven by a need for new ways to investigate nanoscale materials, such as nanoparticles (Fig. 4), nanowires and nanotubes, interfaces between materials, and details of surface processes. XHR SEM offers new and different information that is often complementary to that obtained by S/TEM, atomic force microscopy (AFM), atom probes and other high-resolution analytical techniques.
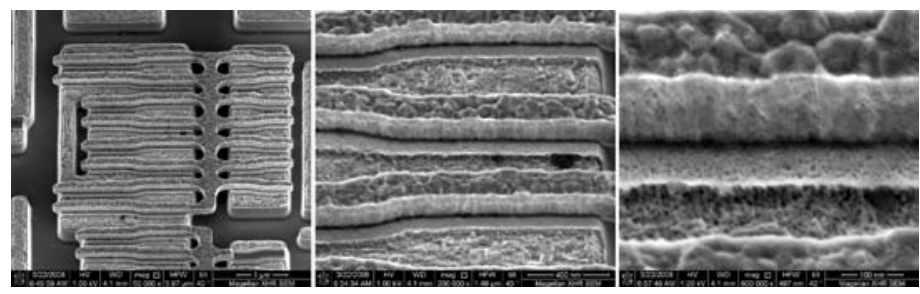

Figure 2: This series of images at increasing magnification demonstrates the Magellan's ability to capture detailed information from complex $3 D$ surfaces at longer, analytical working distances. Smallest HFW: $500 \mathrm{~nm}$.
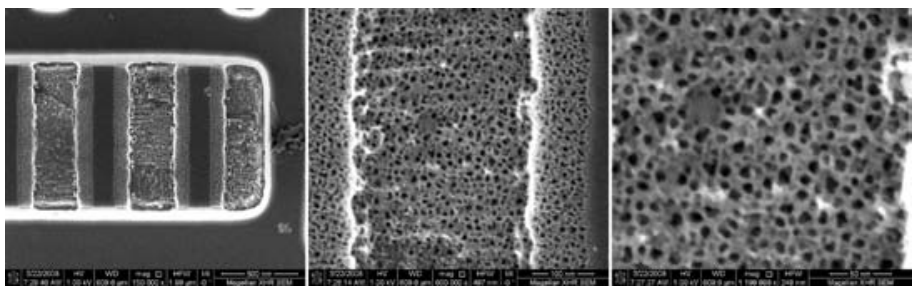

Figure 3: This magnification series, taken from a top down perspective, demonstrates excellent resolution at $1 \mathrm{kV}$ at magnifications impossible on current SEMs. Smallest HFW: $250 \mathrm{~nm}$.

\section{Low Voltage Performance}

Resolution in an SEM is controlled ultimately by the size of the excitation volume from which the imaged signal originates. This, in turn, is determined by the size of the spot formed where the beam intersects the sample surface, and the distance beam electrons penetrate into the sample as they spend their energy in signal-generating interactions with local atoms. Beam penetration is easily reduced by decreasing beam energy (Fig. 5), but in a conventional SEM, the 


\section{Explore Further.}

In 1989, FEl introduced the world's first ESEM $^{\mathrm{m}}$ Environmental SEM.

In 1993, FEl introduced the world's first DualBeam $^{\text {m }}$ FIB/SEM.

In 2005, FEl introduced the Titan ${ }^{\text {tm }}$ TEM, the world's most powerful commercially-available electron microscope.

Now, FEl introduces the Magellan ${ }^{\text {m }}$ family, the world's first Extreme High Resolution SEMs.

Prepare to see things you've never seen before. Visit FEI Company at Microscopy and Microanalysis and learn how you can explore further with this new class of XHR SEM.

See more at fei.com/magellan
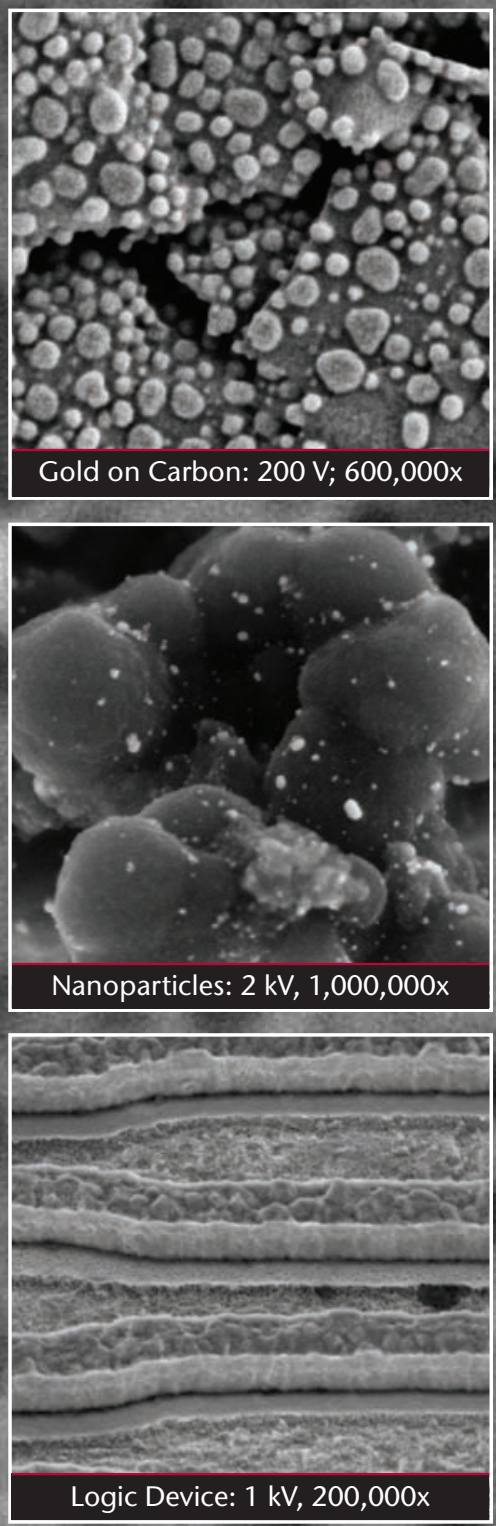

Background image: logic device, 1 kV, 1,200,000x. Logic device images courtesy

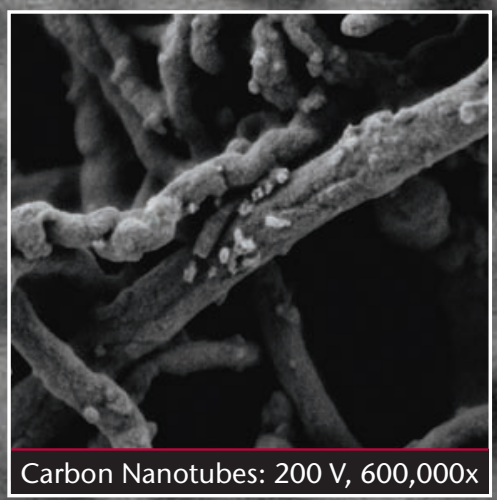

FEI Company

Booth 528

Microscopy and Microanalysis Albuquerque, NM 


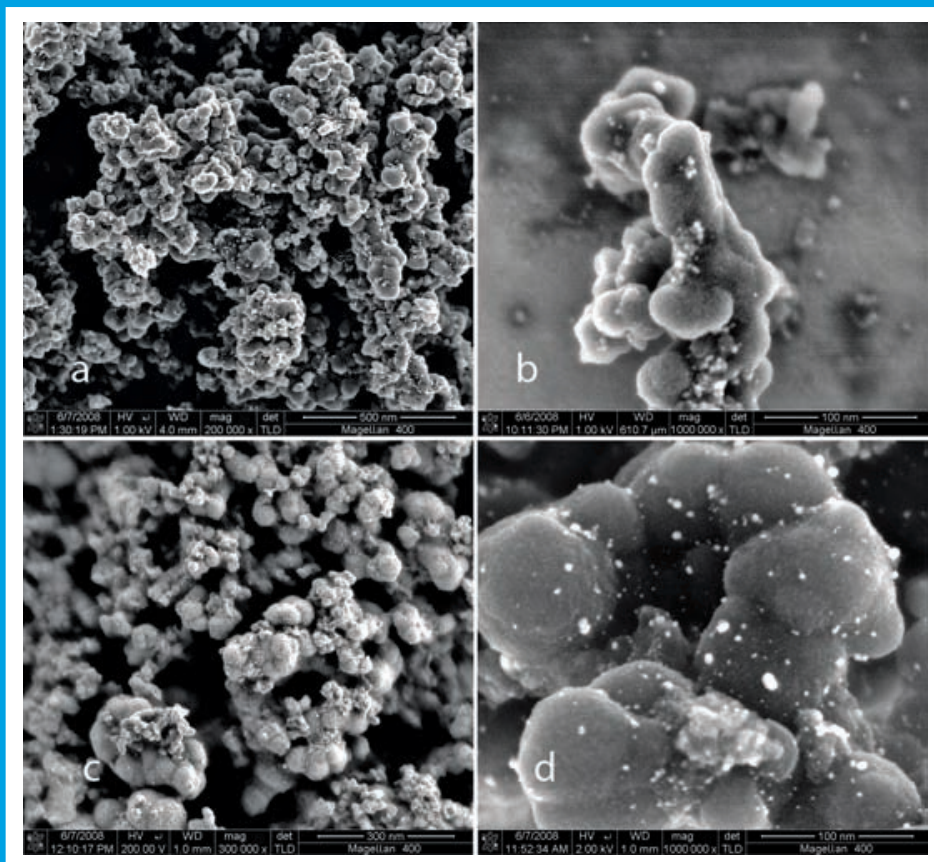

Figure 4: This series of images illustrate the XHR SEM capabilities of the Magellan on Pt catalyst nanoparticles. Magellan allows the jump from traditional UHR magnifications (a) to a million plus magnification (b) at $1 \mathrm{kV}$, where individual nanoparticles are nicely visible. Highly-resolved images are obtained both at very low energies for highly sensitive surface information $(c)$, and very balanced material and topographic information (d).

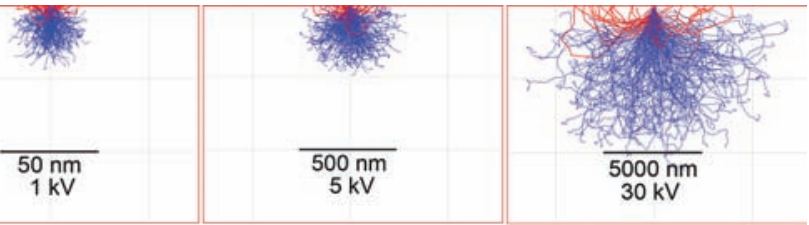

Figure 5: Monte Carlo simulations of electron scattering illustrate the effect of beam energy on interaction volume (the primary electrons are shown in blue, with back-scattered electrons in red)[1]. The effect of interaction volume on resolution depends on many factors, such as the signal chosen for imaging. For example, although the secondary electron signal usually selected for high-resolution imaging originates primarily from within the beam spot, its resolution is degraded by contributions from type II secondary electrons that are created when backscattered beam electrons exit the specimen surface and may originate from a much larger region roughly corresponding to the size of the interaction volume.

gain from reduced penetration is negated by increased spot size at beam energies below a few $\mathrm{keV}$.

The deterioration in spot size at low energy results from chromatic aberration in the lenses used to focus the electron beam [2]. It causes electrons of different energies to focus at different distances-entirely reasonable since the focusing force exerted on the electron by the magnetic field of the lens is directly proportional to the electron's velocity. The effect of chromatic aberration on spot size is determined by the fractional energy spread-a measure of the variability among individual electron energies relative to overall beam energy. Even a few eV of variability has relatively little effect on the trajectories followed by $30 \mathrm{keV}$ electrons through the magnetic field of the focusing lens. But the same level of variability becomes increasingly significant as beam energy decreases, eventually limiting the minimum size of the spot into which the electrons can be focused when balanced against the beam diameter resulting from diffraction through the beam limiting aperture.

The tremendous potential for improved performance over a broad range of SEM applications that would accrue from a break- through in low-voltage resolution became a primary driver in the Magellan development program, and is a cornerstone in the definition of the new XHR performance category the system has established.

The system's low-voltage solution begins with fundamental choices about the lens configuration and electron source. The adverse effects of chromatic aberration can be reduced by decreasing the focal length of the lens (for an electromagnetic lens that means increasing the lens current / field strength / optical power). In a typical, conically shaped SEM objective lens, the focusing field lies enclosed within the lens and the minimum usable focal length corresponds to the minimum distance from the pointed tip of the lens to the specimen. This distance defines the minimum focal length at which the lens can operate (i.e. the closest the specimen can come to the field) SEM objective lens performance is physically limited by the construction of the lens. An SEM optimized for low voltage performance needs to be able to operate at the shortest possible focal length. Generally, this requires a type of lens known as an immersion lens: the sample is immersed in the lens field, which is external to the lens structure. A variety of lens designs are in use, some of which can be operated in either conventional or immersion mode. It is important that the lens assembly does not restrict the flexibility and broad applicability that are fundamental to SEM's enduring value and popularity. The XHR SEM combines immersion capability with a highly flexible lens and chamber configuration that still allows for large or multiple samples, and a wide range of sample tilt angles.

The choice of electron source also affects low-voltage performance. For example, a Schottky field emission source provides a fivefold reduction in energy spread $(0.6-1.0 \mathrm{eV})$ compared to a tungsten filament, as well as an immediate and commensurate reduction in the minimum useful accelerating voltage (where chromatic aberration begins to limit performance) to a few hundred volts. Cold-field emitters have an even narrower energy distribution $(0.25-0.35 \mathrm{eV})$ but that benefit is offset by their instability, limited current capacity, high-vacuum requirements, vulnerability to contamination, and need for frequent "flashing" to restore the condition of the emitter tip.

The most innovative aspect of the electron column is its patented UniColore (UC) technology. In UC mode the beam is energy dispersed and focused through a small aperture that eliminates the most extreme components of the energy distribution, resulting in a beam with an energy spread of less than 0.2 eV. Figure 6 shows a schematic representation of how an improved energy spread in UC mode is balanced against diffrac-

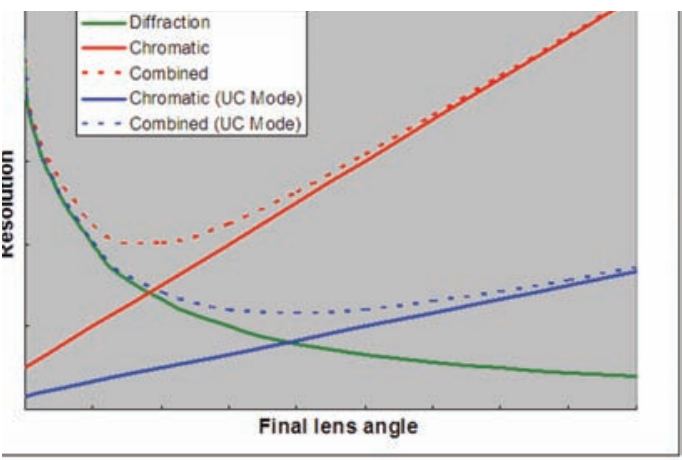

Figure 6: Low voltage spot size is limited primarily by diffraction and chromatic aberration. This schematic figure illustrates the effect on this balance of reducing the energy spread by using UC mode. The minimum spot size is reduced and occurs at a larger lens angle (larger aperture/more beam current). Note: the scales of both axes are linear, with arbitrary units. 


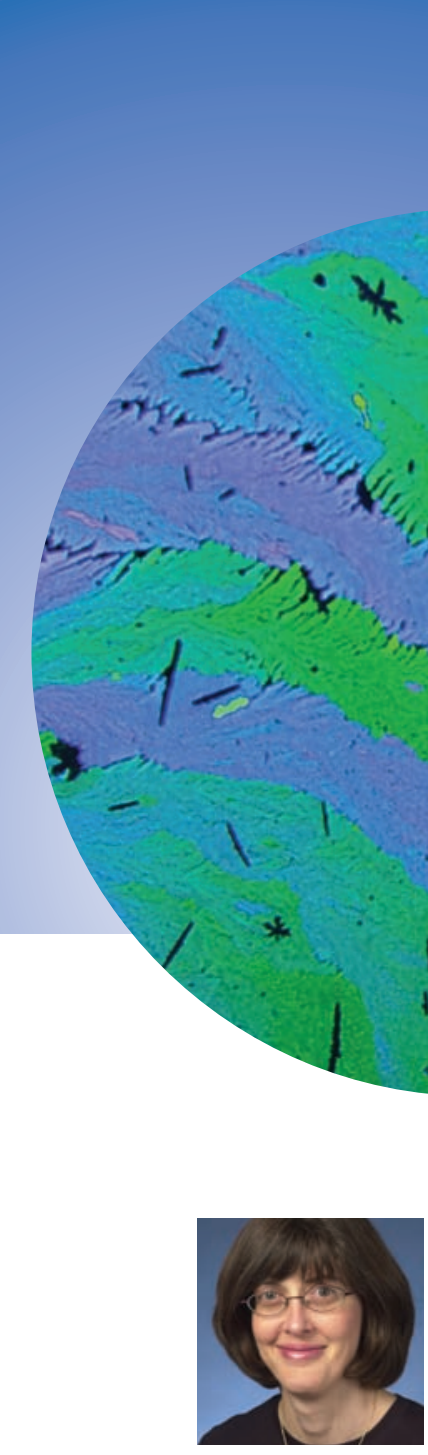

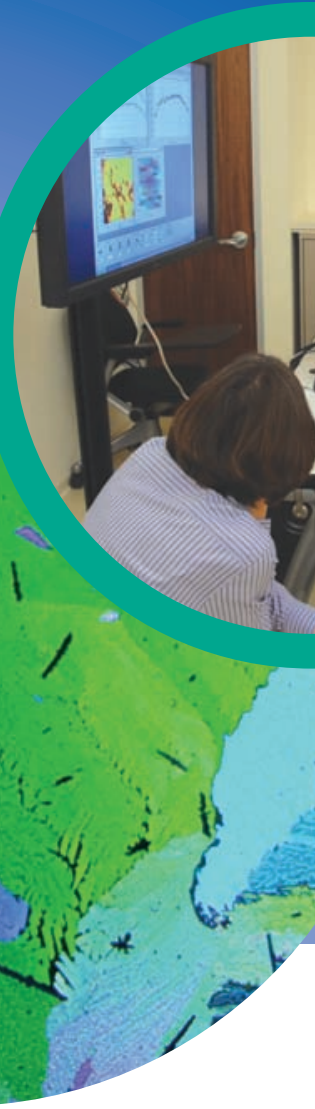

Learn from McCrone scientists with over $\mathbf{5 0}$ years of combined experience.

The College of Microscopy courses in Infrared and Raman Microscopy are designed to provide practical instruction in "real world" use of the FTIR and Raman microscopes. The classes utilize demonstrations and laboratory exercises supplemented with lectures. Students are strongly encouraged to bring their own samples for analysis.

Gretchen-Senior Research Chemist with McCrone Associates since 1995, Ph.D. in Archaeological Chemistry from the University of London, with a fellowship at the Metropolitan Museum of Art, she is responsible for chemical analysis using infrared spectroscopy, Raman microspectroscopy, gas and liquid chromatography, polarized light microscopy and microchemical techniques. She has problem-solving experience in a wide variety of industries including pharmaceutical, paints and coatings, electronics, forensics, polymers and metals.

Kate-Ph.D. in Physical Chemistry from Loyola University of Chicago, with McCrone Associates since 2004, employs infrared and Raman spectroscopy, gas and liquid chromatography, light microscopy and wet chemical methods in the course of analytical problem-solving for a wide variety of clients. At Unilever/Helene Curtis, Inc. she used spectroscopic techniques to support product claims, conduct ex-vivo and in-vivo performance testing; and assist manufacturing facilities in the development and production aspects of a variety of personal care products.

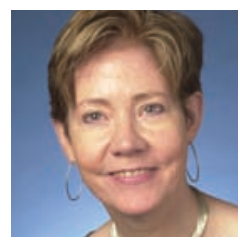

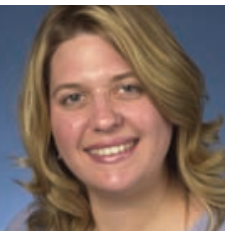

Kristie - Research Microscopist with McCrone Associates since 1998, at Rush University Medical Center in Chicago she gained microscopy experience in a cancer research laboratory and was certified as a Medical Technologist in Immunology. Current work involves microscopical analysis of particles in micrometer and sub-micrometer size ranges including: manipulation, characterization and preparation for instrumental ultramicroanalysis. Current research interests have focused on glass delamination in pharmaceutical products.

Mary-M.S., Analytical Chemistry, Loyola University, Chicago, 20 years of consulting and analytical service experience with particular specialization in plastic and polymer analysis, and currently a Senior Research Chemist at McCrone Associates. Served as VP of Analytical Services for Bodycote Broutman, Inc., a consulting company specializing in contract testing and R\&D for the plastics industry.

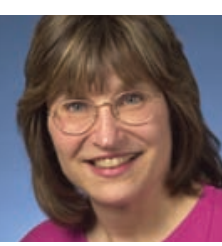




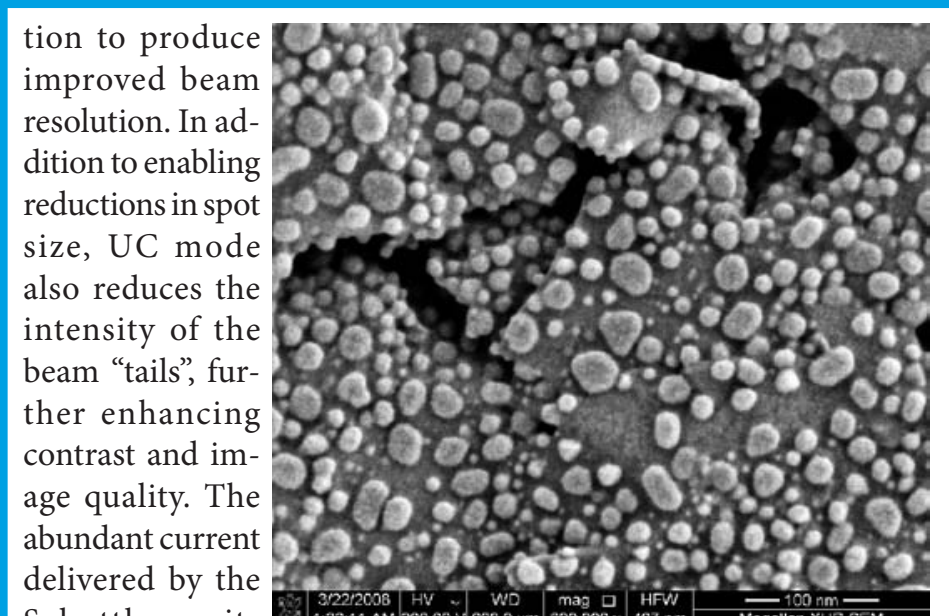

Schottky emit-

ter and the effi-

cient design of

the gun ensure

ample current in

UC mode for the
Figure 7: Au-C sample at $200 \mathrm{eV}$ landing energy using $1 \mathrm{kV}$ of beam deceleration. Under these conditions the $A u$ and $C$ contrast levels are similar to each other, quite different from the result normally seen at $1 \mathrm{kV}$ accelerating voltages and above (see text for more details). high resolution imaging applications it is intended to support. Ultimately, UC mode results in a beam that has an energy spread less than that of a cold field emitter but still retains the robust stability and other benefits of the Schottky source. In addition, UC mode is implemented as a simple push button operation and so its benefits are gained without the complexity of aberration correctors or similar approaches to performance improvement [3]. In high-current applications, UC mode is automatically switched off, instantly restoring the gun to its normal operating capability.

\section{Traditional SEM Capability}

While extending SEM imaging capability into the XHR realm, the Magellan sacrifices none of the traditional advantages of SEM in speed, flexibility, ease-of-use, minimal sample preparation, analytical capability, prototyping and much more. Its large specimen chamber easily accommodates a fully-automated 5-axis stage offering $0.5 \mu \mathrm{m}$ precision over $100 \mathrm{~mm}$ of $x / y$ travel. The accuracy of the stage's piezoceramic drives is a critical component of the eucentric tilt capability, allowing users to explore different viewing perspectives quickly without losing sight of the object. Its electrostatic scanning is fast and accurate at all beam currents and accelerating voltages and eliminates the hysteresis inherent in scanning coils. Provision for a beam blanker at a fixed cross over position enables fast blanking without tailing or streaking for lithographic and nanoprototyping applications.

A variety of detectors is available to support complex imaging operations. Secondary detection for topographic contrast and high-resolution applications is provided by an in-chamber Everhart-Thornley detector and a highly efficient in-lens detector. An in-lens backscatter mode and a solid state backscattered electron detector mounted under the pole piece provide compositional ( $z$-contrast) imaging. A unique low voltage, high contrast detector (vCD) designed to increase detection efficiency for the low-energy backscattered electron generated by low-voltage microscopy is particularly useful in conjunction with beam deceleration to tune image contrast for specific applications.

Beam deceleration, which applies a negative bias to the specimen, permits imaging with beam landing energies (accelerating voltage minus decelerating voltage) as low as $50 \mathrm{eV}$. The decelera- tion field acts as an additional electrostatic lens, reducing spot size further - with the improvement in spot performance becoming greater as the landing energy is reduced [4]. The decelerating bias can also be used to enhance and manipulate image contrast by accelerating secondary and backscattered electrons toward the vCD detector.

Figure 7 shows an Au-on- $\mathrm{C}$ image taken using UC mode at a beam landing energy of $200 \mathrm{eV}$ ( $1 \mathrm{kV}$ of beam deceleration). It is observed that at $200 \mathrm{eV}$ the gold and carbon contrast levels are much more similar to each other than is the case at $1 \mathrm{keV}$ and above (compare to Fig. 1, where the gold signal is much greater than the carbon one). This change in contrast hints at the valuable image information that can be obtained by observing the same sample under a variety of beam energy conditions at high resolution.

The best optical design in the world cannot deliver its promised performance unless the supporting platform provides sufficient internal stability and resistance to external interferences. As the maker of Titan TEMs, the world's most powerful commerciallyavailable microscopes, FEI probably understands these platform requirements better than most. In fact, the Magellan uses the same constant power lens technology developed for the Titan TEM, which permits changes in the electron optical power of the lens without changing the power dissipation of the lens, a change that might otherwise bring work to a halt while the system came to a new thermal equilibrium. Electron microscopes can be exquisitely sensitive vibration detectors and the most solid column in the world is worth little if the stage and sample are dancing around beneath it. The system's 5-axis piezoceramic stage combines rock solid stability with 0.5 micrometer positioning precision over $100 \mathrm{~mm}$ of travel. The column is also double shielded to reduce the effects of external sources of electromagnetic radiation on the electron beam.

The new XHR SEM is designed for optimal throughput and productivity with an optional automated loadlock. The large stage and flexible sample holders can accommodate up to 40 cleaved cross section samples at a time. An integrated plasma cleaner and liquid nitrogen cold trap maintain pristine conditions in the sample chamber and reduce the effects of sample generated contamination on image quality.

\section{Conclusion}

XHR SEM takes current high-resolution SEM applications and pushes them into a new regime of sub-nm resolution at low beam energies. For semiconductor and data storage manufacturers, this means continuing to use SEM for more node shrinks than was previous thought possible. For the researcher, it opens up exciting imaging possibilities for nanoscale structures, providing new and complementary information to that obtained by S/TEM, AFM and similar techniques.

\section{References}

[1] Casino Software: http://www.gel.usherbrooke.ca/casino/

[2] See for example: J. Goldstein et al, Scanning Electron Microscopy and X-Ray Microanalysis., Third Edition. 2003, New York: Plenum.

[3] A. Braun, Semiconductor International (December 2007)

[4] I. Müllerová et al, Surface Science 601 (2007) 4768

\section{Acknowledgements}

The authors gratefully acknowledge the support of the Smart Mix Programme of the Netherlands Ministry of Economic Affairs and the Netherlands Ministry of Education, Culture and Science. Samples shown in Figs. 2 and 3 are courtesy of STMicroelectronics Malta/Grenoble. 


\section{Polishing Matters!}

Flat Lapping \& Polishing

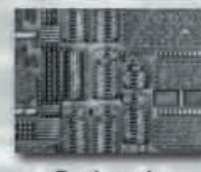

De-layering

- Optical alignment makes flatness set-up

fast \& accurate

- Wide range of sample holders

- All polishing methods
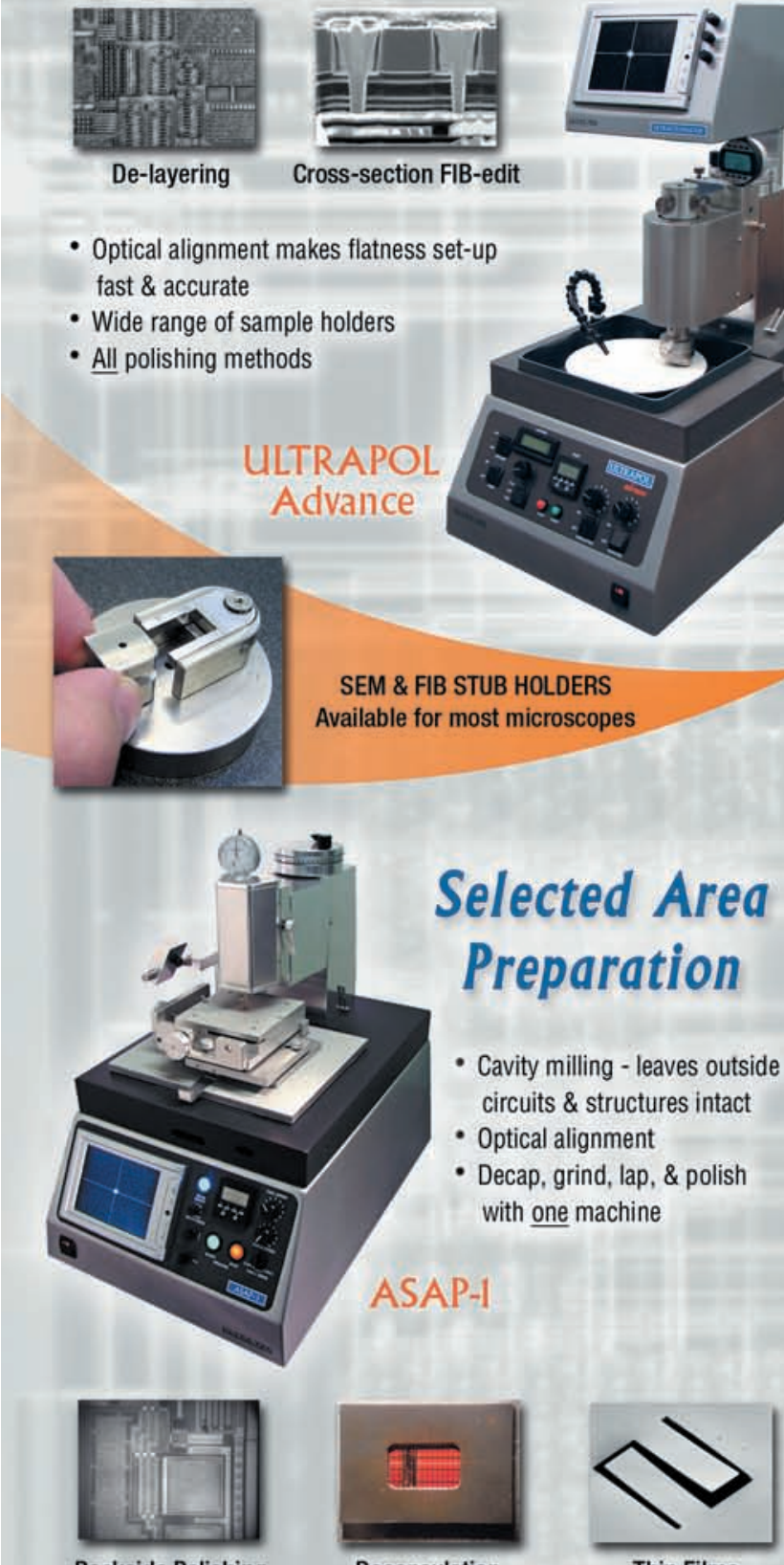

ULTRAPOL Advance

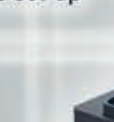

a
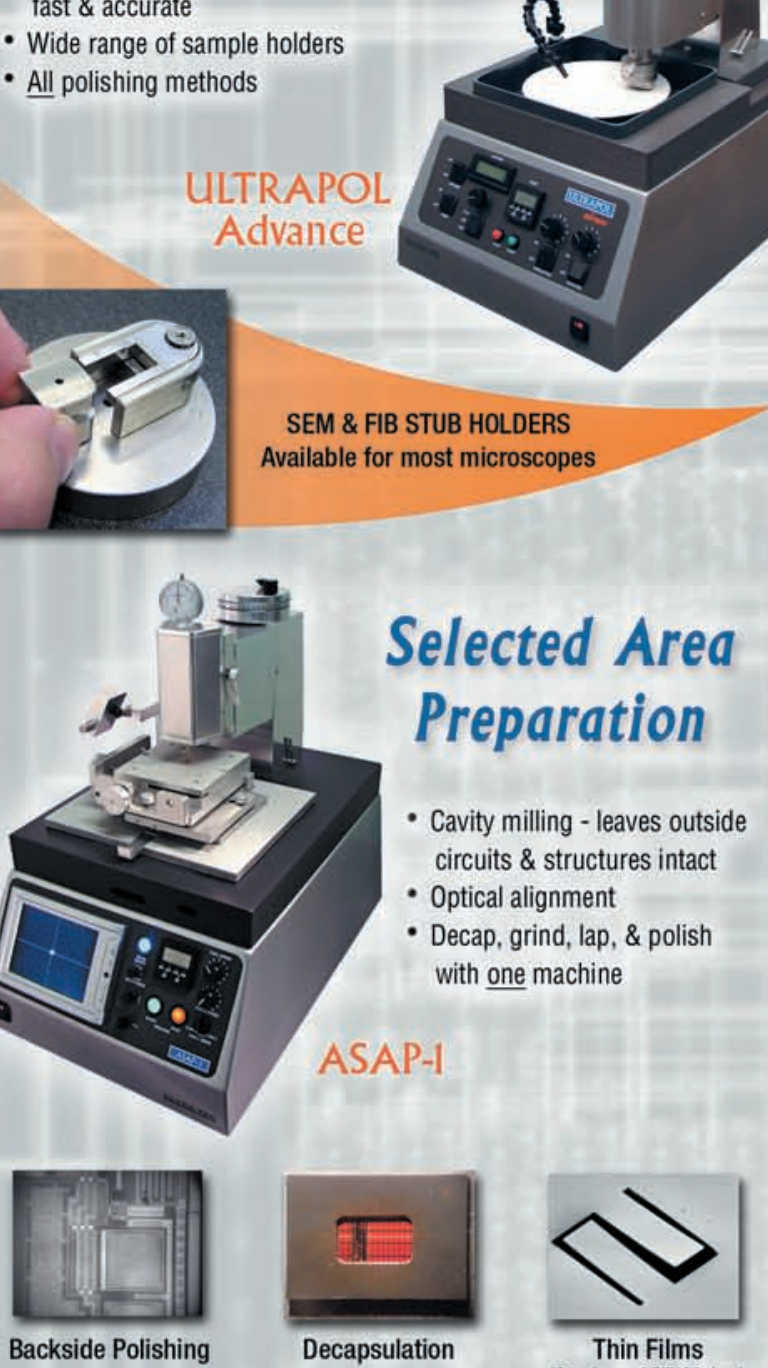

SEM \& FIB STUB HOLDERS Available for most microscopes
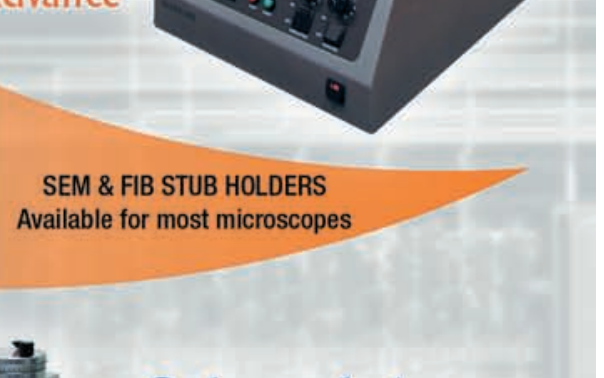

\section{Selected Area}

- Cavity milling - leaves outside circuits \& structures intact

- Optical alignment

- Decap, grind, lap, \& polish with one machine

\section{ASAP-1}

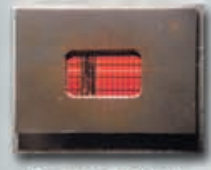

Decapsulation

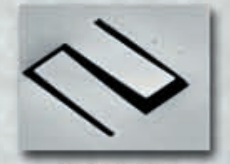

Thin Films (Courtesy of FIBICS, Inc.)

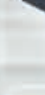
Preparation

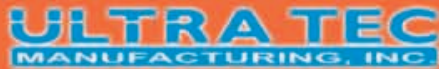

Toll Free (US) 1-877-542-0609

Tel: 1-714-542-0608 Fax: 1-714-542-0627

E-mail: info@ultratecusa.com www.ultratecusa.com

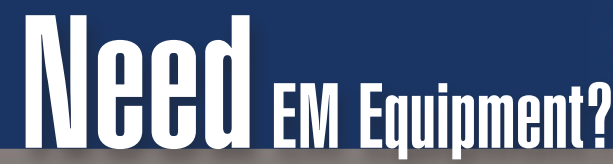

\section{Come to the Expepts.}

Micro-Materials
Hitachi High-Te

ค. (ด) Omniprob

40

Left Coast Instrument Imago Millbroc

XEI Scientific, Inc.

- Represents leading manufacturers

- Supports the Eastern US and Canada

- Offers over 40 years of experience and market knowledge

Need east coast representation?

Have Angstrom Scientific grow your business.

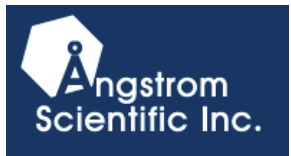

Angstrom Scientific, Inc. (201)-760-2524

Info@angstrom.us

www.angstrom.us
Angstrom Scientific has partnered with leading manufacturers to deliver the right EM equipment solution for your application.

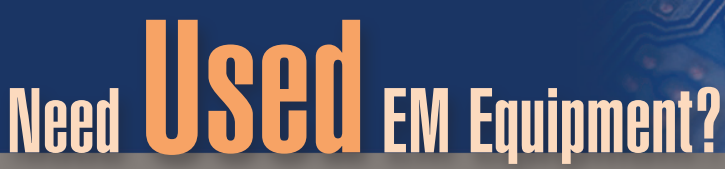

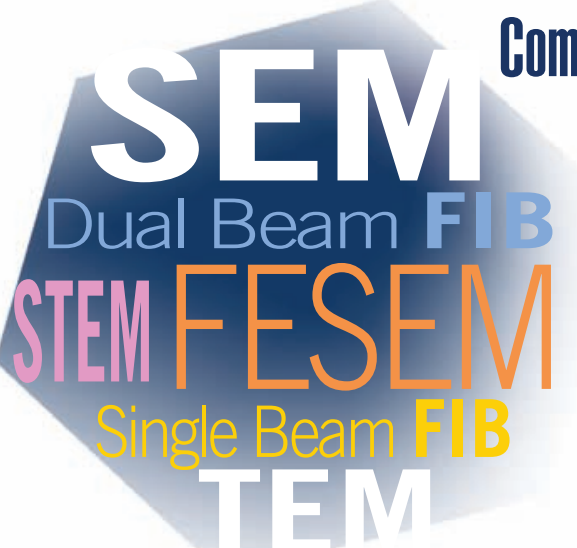

Whether you're looking to buy used EM equipment or have used equipment to sell, Angstrom Scientific is your source.

- Turnkey installation that is guaranteed operational

- All major SEM, TEM, STEM and FIB brands

- 40 years of experience and market knowledge See our current inventory of used EM equipment at www.angstrom.us

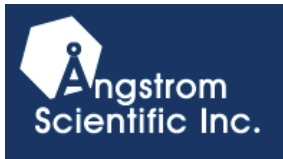

Angstrom Scientific, Inc. (201)-760-2524 Info@angstrom.us www.angstrom.us 